\title{
Selective ablation of carious lesions using an integrated multispectral near-IR imaging system and a novel 9.3- $\mu \mathrm{m}$ CO2 laser
}

Kenneth Chan, Nathaniel Fried, Daniel Fried

Kenneth H. Chan, Nathaniel M. Fried, Daniel Fried, "Selective ablation of carious lesions using an integrated multispectral near-IR imaging system and a novel 9.3- $\mu \mathrm{m}$ CO2 laser," Proc. SPIE 10473, Lasers in Dentistry XXIV, 104730E (8 February 2018); doi: 10.1117/12.2296025 


\title{
Selective Ablation of Carious Lesions using an Integrated Near-IR Imaging System and a Novel 9.3- $\mu \mathrm{m} \mathrm{CO}$ Laser
}

\author{
Kenneth H. Chan ${ }^{1}$, Nathaniel M. Fried ${ }^{2}, \mathrm{PhD}$, and Daniel Fried ${ }^{1}, \mathrm{PhD}$ \\ ${ }^{1}$ University of California, San Francisco, San Francisco, CA 94143-0758 \\ ${ }^{2}$ University of North Carolina at Charlotte, Charlotte, NC 28223
}

\begin{abstract}
Previous studies have shown that reflectance imaging at wavelengths greater than $1200-\mathrm{nm}$ can be used to image demineralization on tooth occlusal surfaces with high contrast and without the interference of stains. In addition, these near-IR imaging systems can be integrated with laser ablation systems for the selective removal of carious lesions. Higher wavelengths, such as 1950-nm, yield higher lesion contrast due to higher water absorption and lower scattering. In this study, a point-to-point scanning system employing diode and fiber lasers operating at $1450,1860,1880$, and 1950-nm was used to acquire reflected light images of the tooth surface. Artificial lesions were imaged at these wavelengths to determine the highest lesion contrast. Near-IR images at 1880 -nm were used to demarcate lesion areas for subsequent selective carious lesion removal using a new compact air-cooled $\mathrm{CO}_{2}$ laser prototype operating at $9.3-\mu \mathrm{m}$. The highest lesion contrast was at $1950-\mathrm{nm}$ and the dual $\mathrm{NIR} / \mathrm{CO}_{2}$ laser system selectively removed the simulated lesions with a mean loss of only $12-\mu \mathrm{m}$ of sound enamel.
\end{abstract}

Keywords: caries removal, SWIR reflectance imaging, image-guided laser ablation

\section{INTRODUCTION}

Studies have shown that near-IR reflectance imaging beyond 1200-nm is well-suited for imaging carious lesions without the interference of stains $[1,2]$. Conventional visible reflectance and quantitative light fluorescence techniques for carious lesion detection yield low lesion contrast and are plagued with poor sensitivity due to stains masking demineralization $[3,4]$. At longer wavelengths the scattering coefficient of enamel decreases, allowing deeper light penetration and decreased backscattered light in sound enamel structure. The scattering coefficient is extremely low beyond $1300-\mathrm{nm}$ and it has not been possible to measure it beyond $1300-\mathrm{nm}$ due to the influence of surface scattering and increased water absorption. Wavelengths coincident with water absorption bands, namely 1450, 1880, and 1950-nm have high water absorption $\left(29,31\right.$, and $113-\mathrm{cm}^{-1}$, respectively) [5]. Interstitial water trapped between enamel prisms and water within the underlying dentin highly absorb these wavelengths and cause sound enamel structure to backscatter less light and appear darker. Therefore, it is likely that longer wavelengths beyond $1700-\mathrm{nm}$ where water absorption increases and light scattering continues to decrease may yield even higher contrast between sound and demineralized enamel.

Lasers are well designed for the selective removal of dental caries lesions. They can be used to precisely remove thin layers of demineralized enamel if a small spot size is used. Studies have demonstrated that $\mathrm{CO}_{2}$ lasers $(\lambda=$ 9.3-9.6- $\mu \mathrm{m}$ ) are ideal for caries removal and have added benefits, namely, rendering treated dental hard tissue with higher resistance to further decay [6]. Typical dental lasers are operated by hand, which nullifies their capacity for high precision and selectivity. Thus, an image-guided computer-controlled laser is ideal for selectively removing caries lesions. Previous work demonstrated that the $\mathrm{CO}_{2}$ laser irradiated enamel does not interfere with the ability to image the tooth surface nor significantly distort the contrast between sound and carious dental hard tissues [7]. Furthermore, in past near-IR image-guided laser experiments, InGaAs cameras were successful in detecting lesion presence and guiding a $\mathrm{CO}_{2}$ laser for lesion removal [8]. However this technique had limitations, it was challenging to precisely align near-IR reflectance images with the laser and 
InGaAs cameras are currently expensive. In this study, we combined the $\mathrm{CO}_{2}$ laser beam with a near-IR thulium fiber laser to improve lesion detection and ablation alignment. A less expensive extended range InGaAs photodiode was used to capture backscattered light at wavelengths beyond $1700-\mathrm{nm}$. This method also is advantageous for improving the signal throughput for improved lesion detection. In this study, we measured the lesion contrast at 1450,1860,1880, and 1950-nm and subsequently evaluated the dual imaging and laser ablation system for removing demineralization from the occlusal surfaces of extracted teeth.

\section{MATERIALS AND METHODS}

\subsection{Sample and Lesion Preparation}

Ten human teeth with non-carious occlusal surfaces were collected and sterilized with gamma radiation. Tooth occlusal surfaces were abraded using air abrasion with $50-\mu \mathrm{m}$ glass beads for 20 seconds to remove all stain and debris from the fissures and remove the outermost fluoride rich layers of enamel to facilitate the demineralization of those surfaces. Next, teeth were mounted in black orthodontic acrylic blocks. Samples were stored in a moist environment of $0.1 \%$ thymol to maintain tissue hydration and prevent bacterial growth. The outlines of a $4 \times 4$ $\mathrm{mm}$ window (roughly 50- $\mu \mathrm{m}$ deep) were cut on the occlusal surfaces of each tooth using a $\mathrm{CO}_{2}$ laser (Impact 2500, GSI Lumonics Rugby, UK) operating at a wavelength of $9.3-\mu \mathrm{m}$, pulse duration of 15 -microseconds and a pulse repetition rate of $5-\mathrm{Hz}$. A water spray was used, and the incident fluence was $170-\mathrm{J} / \mathrm{cm}^{2}$ with a laser pulse diameter of $150-\mu \mathrm{m}$. This served as a reference point for demarcating the treatment region and optical coherence tomography analysis. The enamel surrounding the $4 \times 4 \mathrm{~mm}$ windows created by the laser was covered with red fingernail polish (Revlon, New York, NY). The varnish was removed using

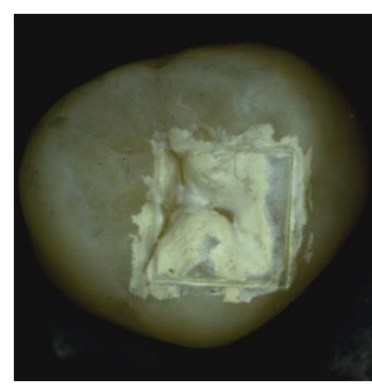

Fig. 1. Depth composition digital microscopy image of artificially generated occlusal lesion using a surface softened lesion model (pH 4.5, 48hrs).

acetone after the lesions were generated. Artificial lesions were created within the $4 \times 4 \mathrm{~mm}$ windows by immersing each tooth into a $50 \mathrm{ml}$ aliquot of a $\mathrm{Ca} / \mathrm{PO}_{4} /$ acetate solution containing $2.0 \mathrm{mmol} / \mathrm{L}$ calcium, 2.0 $\mathrm{mmol} / \mathrm{L}$ phosphate, and $0.075 \mathrm{~mol} / \mathrm{L}$ acetate maintained at $\mathrm{pH} 4.5$ and a temperature of $37^{\circ} \mathrm{C}$ for 48 hours (see Fig. 1). Using cross polarized optical coherence tomography, we identified and quantified lesions roughly between 50-150- $\mu \mathrm{m}$ deep with intact surfaces [9-14].

\subsection{Integrated $\mathrm{CO}_{2}$ Laser and Near-IR Point-to-Point Imaging System}

A diagram of the system is shown in Fig. 2. An RF-excited laser, DL500 prototype from Access Laser Co (Everett, WA) operating at a wavelength of $9.3-\mu \mathrm{m}$ was used with a pulse duration of $25-\mu \mathrm{s}$ and a pulse repetition rate of $200-\mathrm{Hz}$. The laser energy output was monitored using a power/energy meter, ED-200 from Gentec (Quebec, Canada). The laser beam was focused to a beam diameter of $250-\mu \mathrm{m}$ using a $\mathrm{ZnSe}$ lens of $100-\mathrm{mm}$ focal length. A razor blade was scanned across the beam to determine the diameter $\left(1 / \mathrm{e}^{2}\right)$ of the laser beam. The incident fluence was $31 \mathrm{~J} / \mathrm{cm}^{2}$. Computer-controlled XY galvanometers 6200HM series with MicroMax series 671 from Cambridge Technology, Inc. (Cambridge) were used to create controlled movements of the samples during near-IR reflectance image acquisition and subsequent laser irradiation. A pressure air-actuated fluid spray delivery system consisting of a $780 \mathrm{~S}$ spray valve, a Valvemate 7040 controller, and a water reservoir from EFD, Inc. (East Providence, RI) was used to provide a uniform spray of fine water mist $(5 \mathrm{ml} / \mathrm{mm})$ onto the tooth surfaces.

Selected wavelengths $(1460,1860,1880$, and 1950-nm) were used to generate point-to-point near-IR reflectance images. These near-IR diodes were combined with the former mentioned $\mathrm{CO}_{2}$ laser beam with a beamsplitter and focused with the same $100-\mathrm{mm}$ focal length $\mathrm{ZnSe}$

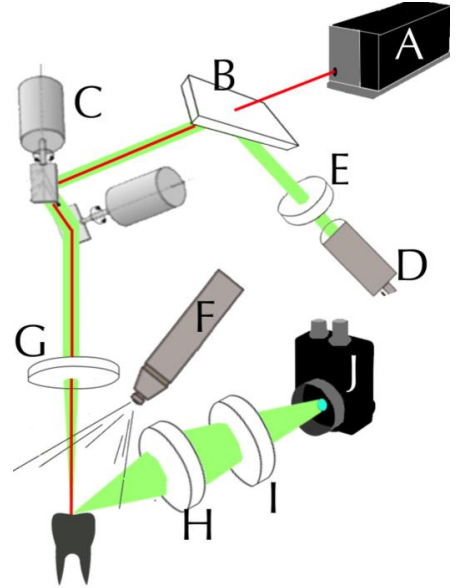

Fig. 2. Combined NIR image-guided caries ablation system: (A) $\mathrm{CO}_{2}$ laser; (B) beamsplitter; (C) galvanometer mirror scanner; (D) near-IR fiber laser (1880-nm) w/ IR lens; (E) collimator; $(F)$ water-/airspray; $(G)$ scanning lens; $(H)$ IR lens; (I) cross-polarizer; (J) near-IR photodetector; (Red line) $\mathrm{CO}_{2}$ laser; (Green line) NIR light. 
lens onto the occlusal tooth surface. Laser light sources used were a 1468-nm superluminescent diode, Model 1480 from Exalos (Langhorne, PA) with a bandwidth of 40-nm and a peak output of 14-mW; a tunable 18501880-nm thulium fiber laser, Model TLT-5 from IPG photonics (Oxford, MA) with an output of 500-mW; and a linearly polarized 200-mW, 1950-nm thulium fiber laser, Model AP-CW from AdValue Photonics (Tucson, AZ).

A near-IR InGaAs photodiode detector, Model PDA10DT from Thorlabs (Newton, NJ) was used to collect backscattered near-IR light from the occlusal surface. Polarizers were placed before the laser sources and the detector to remove specular reflection from the tooth surfaces. Unfortunately, the extinction ratio for our polarizers was not high enough to completely remove all specular reflection at 1950-nm. Therefore for imageguided ablation we choose to use 1880-nm as opposed to 1950-nm, even though 1950-nm yielded higher contrast.

\subsection{Digital Microscopy}

Tooth surfaces were examined after laser irradiation using an optical microscope- VHX-1000 from Keyence (Elmwood, NJ) with a VH-Z25 magnification lens. Depth composition digital microscopy images (DCDM) were acquired by scanning the image plane of the microscope and reconstructing a depth composition image with all points at optimum focus for displaying a fully focused $2 \mathrm{D}$ image. Images of the samples were acquired before and after ablation at 50x magnification.

\subsection{PS-OCT System (OCT)}

An all-fiber-based optical coherence domain reflectometry (OCDR) system with polarization maintaining (PM) optical fiber, high-speed piezoelectric fiber-stretchers and two balanced InGaAs receivers from Optiphase, Inc., (Van Nuys, CA) was used. This two-channel system was integrated with a broadband superluminescent diode (SLD) from Denselight (Singapore) and a high-speed XY-scanning system with a ESP 300 controller and 850GHS stages from Newport (Irvine, CA) for rapid in vitro optical coherence tomography acquisition. The spectral output of the 15-mW SLD was centered at $1317 \mathrm{~nm}$ with a spectral bandwidth full-width at half-maximum (FWHM) of $84 \mathrm{~nm}$. This configuration provided a lateral resolution of approximately $20 \mu \mathrm{m}$ and an axial resolution of $10 \mu \mathrm{m}$ in air. The system is described in greater detail in reference [15]. PS-OCT was used to assess the presence of occlusal lesions and volume of tissue removed by the laser.

\subsection{Imaging, Processing, and Caries Removal} The algorithm used for image-guided removal is shown in Fig. 3. Each step of the algorithm is discussed below.

Samples were air-dried for $\sim 10$ seconds prior to near-IR point-to-point reflectance image acquisition of the tooth occlusal surface. Images were acquired over an area $13 \times 12-\mathrm{mm}$ with a 50 $\mu \mathrm{m}$ pixel pitch. The backscattered near-IR intensity at each point was averaged over 5

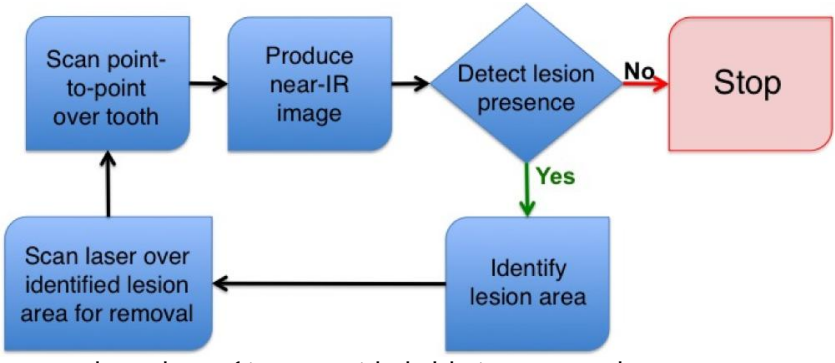

Fig. 3. Flow-chart of image-guided ablation protocol. acquisitions. Each point was rasterized into an 8-

bit near-IR image. Highly scattering demineralized surfaces confer higher signal or appear brighter in the nearIR reflectance images, while sound tissues appear dark (see Fig. 6A-D).

Lesion presence was automatically determined through analysis of the pixel intensity. Pixel intensities were analyzed through an Otsu's adaptive thresholding method (6 cluster groups). This automatically determines an intensity threshold by calculating the median intensity of each cluster and correlating the distance of each point's intensity to its respective clustering group. This method then groups pixels of similar intensity together (see Fig. $6 \mathrm{E}-\mathrm{H})$. As the lesion is removed, clustering becomes overly sensitive, therefore the initial near-IR image was appended to the newly acquired scanned images during lesion removal. The lesion pixels in the new image that are grouped with the lesion pixels in the initial image were subjected to laser treatment. A highly scattering reference target was scanned along with the sample as a reference of maximum signal from scattering so that sequential near-IR reflectance images can be normalized and compared with each other. Healthy sound enamel structure outside the artificially generated lesion was imaged to provide a positive control of sound tissue pixel 
intensity. A look-up-table (LUT) was created which maps out lesion areas demarcated for laser treatment (see Fig. 6I-L).

Using the acquired LUT, the $\mathrm{CO}_{2}$ laser was scanned over the identified lesion area for removal. The distance between laser pulses was $50-\mu \mathrm{m}$, roughly $1 / 5$ of the $250-\mu \mathrm{m}$ laser spot diameter. Repeated serial NIR images and LUTs were taken (Fig. 6) until the lesion was no longer discriminated. All image analysis was carried out using Labview from National Instruments (Austin, TX).

\subsection{Lesion Contrast Analysis}

Ten artificially generated occlusal lesions were imaged with the point-to-point scanning system. Samples were air-dried for $\sim 10$ seconds prior to image acquisition. Each image was scanned with a scattering reference, $\mathrm{BaSO}_{4}$, and normalized for comparison. For each sample and wavelength, the entire $4 \times 4-\mathrm{mm}$ window was summed and averaged to quantify the lesion intensity $\left(\mathrm{I}_{\mathrm{L}}\right)$. A fixed $2 \times 2-\mathrm{mm}$ region-of-interest was selected outside the $4 \times 4-\mathrm{mm}$ fiduciary window at each wavelength to calculate each sample/wavelength's mean sound intensity $\left(\mathrm{I}_{\mathrm{S}}\right)$.

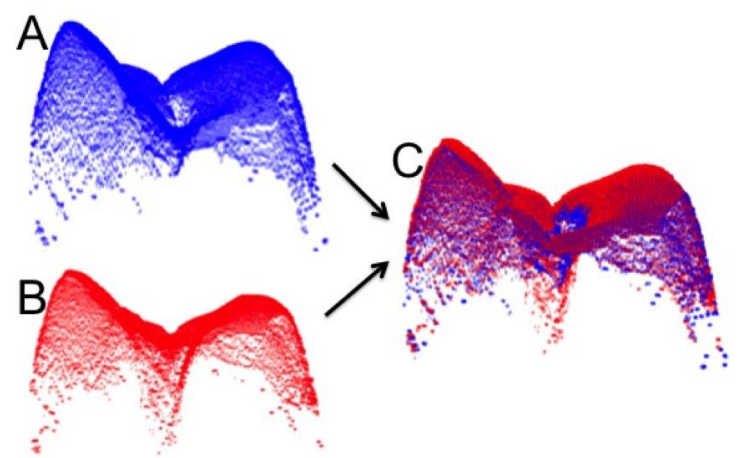

Fig. 4. 3D surfaces imaged with co-polarization OCT. (A) 3D preablation surface (B) 3D postablation surface (C) Pre-/postablation surface $3 \mathrm{D}$ registered to determine volume of tissue removed.

Image contrast was calculated using $\left(\mathrm{I}_{\mathrm{L}}-\mathrm{I}_{\mathrm{S}}\right) / \mathrm{I}_{\mathrm{L}}$. The

image contrast varies from 0 to 1 with 1 being very high contrast and 0 being no contrast. Repeated measures analysis of variance (RM-ANOVA) followed by the Tukey-Kramer post hoc multiple comparison test was used to compare image contrast across each wavelength. All quantitative analysis was done with IgorPro from Wavemetrics (Portland, OR) and Prism from GraphPad (San Diego, CA).

\subsection{Selective Lesion Removal Analysis}

Teeth were imaged before and after image-guided ablation with PS-OCT and digital microscopy (DCDM). DCDM images were visually assessed for lesions before ablation and their removal after ablation. Initial and post-ablation cross-polarized OCT (CP-OCT) scans, were assessed for lesion volume. The automated 3D lesion detection method is based on previous work and uses an automated edge detection routine to extract lesion volume $[11,16]$. Additionally, co-polarization OCT scans were used to quantify laser ablation volume. These copolarization scans were processed to extract the occlusal surface topography (Fig. 4A \& B). Background subtraction was carried out by subtracting the mean with 5 standard deviations of the background that was selected from the top 10 pixels of $220 \mathrm{~b}$-scans. These data points provided consistent background signal intensity and manifested a standard deviation less than $0.04 \mathrm{~dB}$. A 50x50x2.56 anisotropic diffusion Gaussian smoothing filter was applied. In addition, a $3 \times 3 \times 3$ rotating kernel transformation (RKT) technique was applied to emphasize lines and eliminate speckle noise. Surfaces were extracted by using a full width half maximum edge detection approach. Initial and post-ablation surfaces were 3D registered using an Iterative Closest Point algorithm (see Fig.4). The $4 \times 4-\mathrm{mm}$ fiduciary window was cropped out before 3D registration to ensure accurate $3 \mathrm{D}$ alignment. A linear interpolator was applied to the post-ablative (moving image) scans to fit the registered scans over the initial (fixed image) scans. Subtraction of the registered post-ablative scans from the initial surface scans was used to quantify ablation volume and process $2 \mathrm{D}$ ablation maps. These $2 \mathrm{D}$ ablation depth maps were compared to initial 2D lesion depth maps to assess selectivity. Furthermore, post-ablative 2D lesion depth maps were analyzed to determine complete lesion removal.

\section{RESULTS AND DISCUSSION}

Images of one of the samples are shown in Fig. 5 for 1450, 1860, and 1880-nm. The highest contrast was at 1950 - $\mathrm{mm}$, however there was contamination of the images from specular reflection. Therefore, we chose 1880$\mathrm{nm}$ to guide the $\mathrm{CO}_{2}$ laser for selective caries removal. 


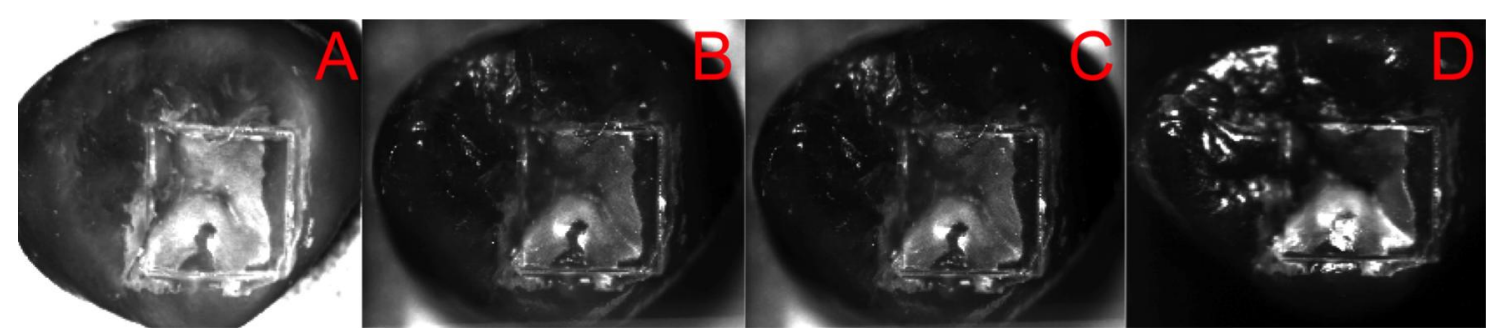

Fig. 5. Near-IR reflectance images generated by the point-to-point scanner. (A) 1450-nm, (B) 1860-nm, (C) 1880-nm, (D) 1950-nm.

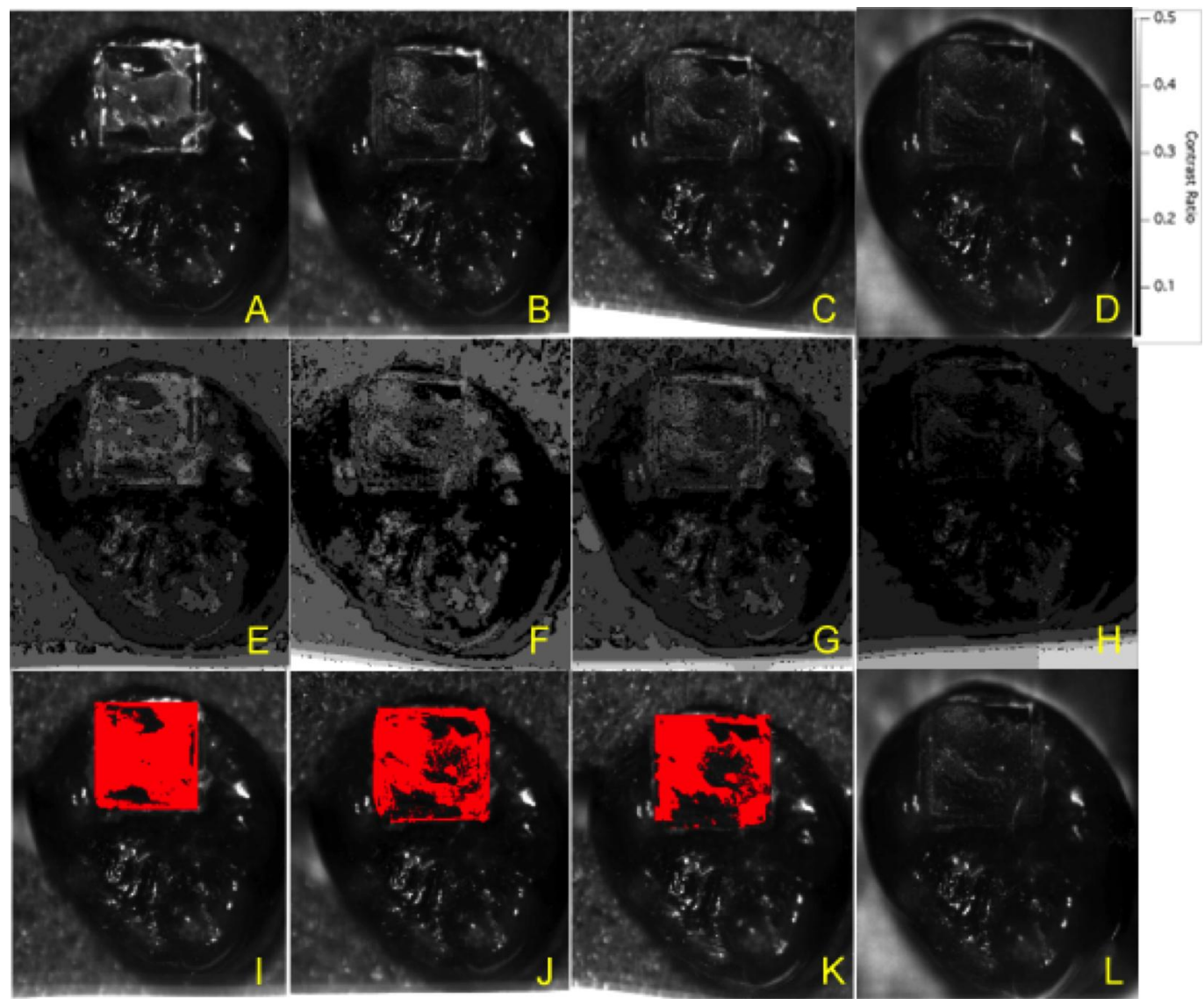

Fig. 6. (A-F) Top row, serially lesion ablated near-infrared (NIR) reflectance images generated from point-to-point scanning. (E-F) Middle row, Otsu's adaptive thresholding clusters group of similar pixel intensities together. (I-L) Bottom row, look-up table (in red) overlaid on top of NIR reflectance images. (A) Initial near-IR point-to-point image of sample. (D) Final near-IR point-to-point reflectance image of sample. After 4 iterations (L), demineralization can no longer be detected, suggesting lesion was completely removed within the $4 \times 4-\mathrm{mm}$ window.

Serial point-to-point near-IR reflectance images and LUTs of the ablation process are shown in Fig. 6. Demineralization appears whiter with higher intensity than sound enamel in these near-IR reflectance images. After four iterations of image-guided demineralization removal, demineralization (white) disappears in the 4x4$\mathrm{mm}$ fiduciary window (Fig. 6D) and the lesion detection algorithm is unable to further identify any lesion area (Fig. 6H \& L). These images demonstrate that a point-to-point scanning system is capable of producing high detail images and the lesion detection algorithm can differentiate lesion and sound tissues. Additionally, the combined near-IR diode/ $\mathrm{CO}_{2}$ laser is correctly aligned since the targeted areas disappear as the removal continues.

PS-OCT images were taken before and after the removal process. The occlusal surface topographies were extracted, 3D registered, and subtracted from one another to produce 2D ablation depth maps (Fig. 7A). In these 

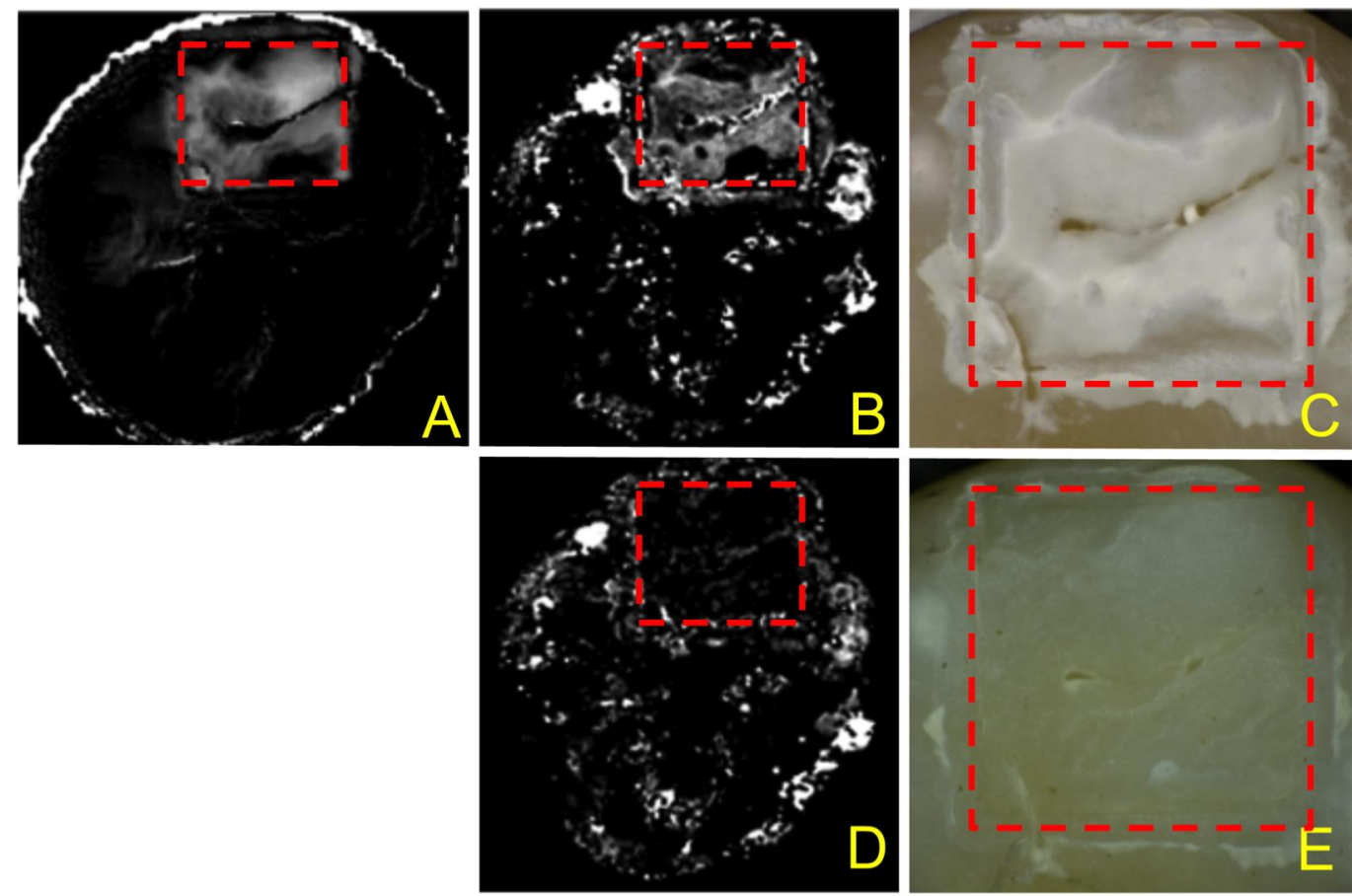

Fig. 7. Assessment of selective caries removal. Red dashed line encircles the $4 x 4-m m$ fiduciary window. (A) 2D ablation depth map created from subtracting initial co-polarization OCT surface scans from registered postremoval scans, (B) Initial 2D lesion depth map, (C) Initial visible image, (D) Post-removal 2D lesion depth map, (E) Post-removal visible image. The $4 \times 4-\mathrm{mm}$ window in the $2 \mathrm{D}$ ablation depth map(A) matches well with the initial 2D lesion depth map(B). The lesion is absent in post-removal 2D lesion depth map(D), indicating lesion removal. Note: Lack of thermal and mechanical damage in visible post-removal.

ablation depth maps, deeper ablation depths appear white. The white circle encircling the $2 \mathrm{D}$ ablation map is the occlusal surface drop off, which is an artifact from registering tilted surfaces. These images visualize the shape and depth of the laser removal.

PS-OCT images were used to assess the lesion depth (Fig. 7B). Deeper lesion depths are indicated by brighter pixels. The 2D ablation depth shape and depth correctly corresponds to the lesion depth map within the reddashed box (Fig. 7A \& B). This demonstrates that the near- $\mathrm{IR} / \mathrm{CO}_{2}$ laser system correctly identified and ablated the lesion within the $4 \times 4-\mathrm{mm}$ fiduciary window.

To ensure the complete lesion removal, post-ablative PS-OCT data were taken and visualized in a 2D lesion depth map (Fig. 7D). The lack of lesion depth (white spots) within the red-dashed fiduciary window indicates that most of the lesion was removed.

Visible depth composition digital images were taken prior to laser treatment (Fig. 7C) and after lesion removal (Fig.7E). Within the fiduciary window of the image prior to removal, a white surface is visible, indicative of a surface lesion (Fig. 7C). After lesion removal, the white surface confined within the fiduciary window is absent and shows a sound enamel surface without any traces of thermal damage (Fig. 7E).

In summary, this study suggests that the enamel scattering coefficient continues to drop beyond $1700-\mathrm{nm}$ and that 1950-nm reflectance imaging yields the highest lesion contrast. Future studies will likely employ this wavelength for lesion detection and selective removal. This study also shows the potential of point-to-point nearIR reflectance imaging for image-guided ablation of carious tissue. The combined near-IR diode and $\mathrm{CO}_{2}$ laser eliminated any alignment errors caused in previous experiments. Additionally, these studies have demonstrated the use of PS-OCT for assessing selective caries removal and nondestructively assessing lesion volume. This technique can be used to further our studies on minimally invasive procedures for dentistry. 


\section{ACKNOWLEDGMENTS}

Access Laser Co. for providing their new compact air-cooled RF-excited 9.3- $\mu \mathrm{m} \mathrm{CO} \mathrm{C}_{2}$ laser. This work was supported by NIH/NIDCR Grants R01-DE019631 and F31-DE026350.

\section{REFERENCES}

[1] S. Chung, D. Fried, M. Staninec et al., "Multispectral near-IR reflectance and transillumination imaging of teeth," Biomed Opt Express, 2(10), 2804-2814 (2011).

[2] W. A. Fried, C. L. Darling, K. Chan et al., "High contrast reflectance imaging of simulated lesions on tooth occlusal surfaces at near-IR wavelengths," Lasers Surg Med, 45, 533-541 (2013).

[3] C. M. Bühler, P. Ngaotheppitak, and D. Fried, "Imaging of occlusal dental caries (decay) with near-IR light at 1310-nm," Opt Express, 13(2), 573-582 (2005).

[4] E. C. Almaz, J. C. Simon, D. Fried et al., "Influence of stains on lesion contrast in the pits and fissures of tooth occlusal surfaces from 800-1600-nm," Proc SPIE, Vol 9692, (2016).

[5] G. M. Hale, and M. R. Querry, "Optical constants of water in the 200-nm to 200- $\mu \mathrm{m}$ wavelength region.," Appl Optics, 12, 555-563 (1973).

[6] N. Konishi, D. Fried, J. D. B. Featherstone et al., "Inhibition of secondary caries by $\mathrm{CO}_{2}$ laser treatment," Amer. J. Dent., 12(5), 213-216 (1999).

[7] N. R. LaMantia, H. Tom, K. H. Chan et al., "High contrast optical imaging methods for image guided laser ablation of dental caries lesions." Proc SPIE, Vol 8929 O1-7.

[8] K. H. Chan, and D. Fried, "Selective removal of demineralization using near infrared cross polarization reflectance and a carbon dioxide laser," Proc SPIE, Vol 8208, (2012).

[9] J. D. Featherstone, R. Glena, M. Shariati et al., "Dependence of in vitro demineralization of apatite and remineralization of dental enamel on fluoride concentration," J Dent Res, 69 Spec No, 620-5; discussion 634-6 (1990).

[10] R. S. Jones, C. L. Darling, J. D. Featherstone et al., "Imaging artificial caries on the occlusal surfaces with polarization-sensitive optical coherence tomography," Caries research, 40(2), 81-89 (2006).

[11] H. Kang, J. J. Jiao, L. Chulsung et al., "Nondestructive assessment of early tooth demineralization using cross-polarization optical coherence tomography," Selected Topics in Quantum Electronics, IEEE Journal of, 16(4), 870-876 (2010).

[12] K. H. Chan, A. C. Chan, W. A. Fried et al., "Use of 2D images of depth and integrated reflectivity to represent the severity of demineralization in cross-polarization optical coherence tomography," J Biophotonics, 8( 1-2), 36-45 (2015).

[13] M. H. Le, C. L. Darling, and D. Fried, "Automated analysis of lesion depth and integrated reflectivity in PS-OCT scans of tooth demineralization," Lasers Surg Med, 42(1), 62-8 (2010).

[14] R. C. Lee, C. L. Darling, and D. Fried, "Automated detection of remineralization in simulated enamel lesions with PS-OCT." Proc SPIE, Vol. 8929, 1-8.

[15] D. Fried, J. Xie, S. Shafi et al., "Early detection of dental caries and lesion progression with polarization sensitive optical coherence tomography," J. Biomed. Optics, 7(4), 618-627 (2002).

[16] R. C. Lee, H. Kang, C. L. Darling et al., "Automated assessment of the remineralization of artificial enamel lesions with polarization-sensitive optical coherence tomography," Biomed Opt Express, 5(9), 2950-62 (2014). 